\title{
Cochrane
}

Library

Cochrane Database of Systematic Reviews

\section{Psychological interventions for depression and anxiety in patients with coronary heart disease, heart failure or atrial fibrillation (Protocol)}

Ski CF, Taylor RS, McGuigan K, Lambert JD, Richards SH, Thompson DR

Ski CF, Taylor RS, McGuigan K, Lambert JD, Richards SH, Thompson DR.

Psychological interventions for depression and anxiety in patients with coronary heart disease, heart failure or atrial fibrillation.

Cochrane Database of Systematic Reviews 2019, Issue 12. Art. No.: CD013508.

DOI: 10.1002/14651858.CD013508.

www.cochranelibrary.com

Psychological interventions for depression and anxiety in patients with coronary heart disease, heart failure or atrial fibrillation (Protocol)

Copyright @ 2019 The Cochrane Collaboration. Published by John Wiley \& Sons, Ltd. 
TABLE OF CONTENTS

HEADER 1

ABSTRACT

BACKGROUND

OBJECTIVES

METHODS

ACKNOWLEDGEMENTS

REFERENCES

ADDITIONAL TABLES

APPENDICES

CONTRIBUTIONS OF AUTHORS

DECLARATIONS OF INTEREST

SOURCES OF SUPPORT 
[Intervention Protocol]

\section{Psychological interventions for depression and anxiety in patients with coronary heart disease, heart failure or atrial fibrillation}

Chantal F Ski ${ }^{1}$, Rod S Taylor ${ }^{2}$, Karen McGuigan ${ }^{1}$, Jeffrey D Lambert ${ }^{3}$, Suzanne H Richards ${ }^{4}$, David R Thompson ${ }^{1}$

1School of Nursing and Midwifery, Queen's University Belfast, Belfast, UK. 2MRC/CSO Social and Public Health Sciences Unit \& Robertson Centre for Biostatistics, Institute of Health and Well Being, University of Glasgow, Glasgow, UK. ${ }^{3}$ College of Medicine and Health, University of Exeter, Exeter, UK. ${ }^{4}$ Leeds Institute of Health Sciences, University of Leeds, Leeds, UK

Contact address: Chantal F Ski, School of Nursing and Midwifery, Queen's University Belfast, 97 Lisburn Road, Belfast, BT9 7BL, UK. c.ski@qub.ac.uk.

Editorial group: Cochrane Heart Group

Publication status and date: New, published in Issue 12, 2019.

Citation: Ski CF, Taylor RS, McGuigan K, Lambert JD, Richards SH, Thompson DR. Psychological interventions for depression and anxiety in patients with coronary heart disease, heart failure or atrial fibrillation. Cochrane Database of Systematic Reviews 2019, Issue 12. Art. No.: CD013508. DOI: 10.1002/14651858.CD013508.

Copyright $@ 2019$ The Cochrane Collaboration. Published by John Wiley \& Sons, Ltd.

\section{A B S T R A C T}

This is a protocol for a Cochrane Review (Intervention). The objectives are as follows:

To assess the efficacy and costs of psychological interventions (alone, or with CR or pharmacotherapy, or both) in adults who have a diagnosis of $\mathrm{CHD}$, HF or AF compared to no intervention, or treatment as usual, on psychological and clinical outcomes. 


\section{B A C K G R O U N D}

\section{Description of the condition}

For the purpose of this Cochrane systematic review, heart disease (HD) will encompass three of the most common cardiovascular conditions: coronary heart disease (CHD), heart failure (HF) and atrial fibrillation (AF). CHD is the number one cause of death, accounting for 9.43 million deaths worldwide in 2016, approximately 4.95 million men and 4.48 million women (WHO 2018). HF and AF are cardiovascular disease (CVD) epidemics with similar underlying risk factors and pathophysiology and increasingly reported incidence (Benjamin 2017; Staerk 2017). People with HD experience substantial burden that includes fatigue, shortness of breath, poor health-related quality of life (HRQoL), increased risk of mortality and hospital admissions, and high healthcare costs (Garster 2009; Gheorghe 2018; Giedrimiene 2017; Long 2019; Schmidt 2016).

Over the past two decades significant reductions in mortality have been achieved in people with HD through pharmacological and device therapy (Anderson 2014; Mensah 2017). Subsequently the focus has shifted towards psychological outcomes (Richards 2018; Wan 2014). Depression and anxiety are highly prevalent in people diagnosed with $\mathrm{CHD}, \mathrm{HF}$ and $\mathrm{AF}$, and result in worse outcomes, such as poorer HRQoL, and increased morbidity and mortality (Dhar 2016; Freedland 2015; Gale 2014). With the aim of improving patient outcomes, psychological approaches tackling stress management are included in cardiovascular prevention guidelines internationally (e.g. BACPR 2017; NICE 2018; Piepoli 2016), although there is a move toward different sorts of targeting of psychological interventions, for example, mood states (Reavell 2018), and adherence to lifestyle change (Khanji 2018), and medicines (Bosworth 2018)

The Cochrane Review, Richards 2017, showed that for people with $\mathrm{CHD}$, psychological interventions did not reduce mortality (from any cause), or the risk of cardiac surgery or of having another heart attack. Psychological interventions were found to reduce the risk of cardiac deaths and participant-reported symptoms of depression, anxiety and stress. However, participants had varying levels of psychopathology, and of the 35 included trials 10 did not report psychological status of participants at baseline despite testing the effectiveness of a psychological intervention. Due to the high proportion of trials having a mixed population (i.e. participants with and without a reported psychological disorder at baseline, and trials not reporting psychological status) the evidence remains equivocal as to whether psychological interventions should solely target people with $\mathrm{CHD}$ and with established psychological disorders as opposed to mixed populations (Richards 2017; Richards 2018). To assist in determining whether those in greatest need benefit most from psychological interventions, the current review will focus on adults with a diagnosis of HD, with and without 'reported' depression and anxiety.

In summary, $\mathrm{CHD}, \mathrm{HF}$ and $\mathrm{AF}(\mathrm{HD})$ are growing global health and economic challenges (Cozzolino 2018; Lesyuk 2018; Shafie 2018). By including the three most prevalent cardiovascular conditions this Cochrane systematic review will extend previous reviews that have focused on specific cardiovascular diagnoses (Carney 2017; Lichtman 2014; Richards 2017; Whalley 2011). Further, comparison of trials of psychological interventions for people with $\mathrm{HD}$, with and without reported depression or anxiety, will assist in determining the possible efficacy in these subpopulations.

\section{Description of the intervention}

This review will include psychological interventions delivered by healthcare workers who have been trained in their delivery. By nature, psychological interventions are varied (i.e. content, composition), ranging from traditional psychological therapies (e.g. cognitive behavioural therapy) used to identify and correct dysfunctional emotions, behaviours and cognitions through a goalorientated, systematic procedure in people with cardiac conditions (Kaplan 2009), to third-wave cognitive behavioural therapies (e.g. mindfulness-based stress reduction).

In 2009, National Institute of Health and Care Excellence (NICE) first published guidelines for effective delivery of psychological interventions for depression among those with chronic health conditions. The guidelines stipulated that interventions of this type be delivered by competent delivery agents or practitioners and advocated a more structured approach to guide interventions (NICE 2009). Accordingly, this review will focus on psychological interventions addressing depression and anxiety in HD delivered by healthcare workers trained in the delivery of the intervention.

Best practice guidelines advocate that people diagnosed with HD are offered cardiac rehabilitation (CR; BACPR 2017; Lichtman 2014; NICE 2018; Piepoli 2016; Ponikowski 2016; Woodruffe 2015). The following definition encompasses the key concepts of CR: "The coordinated sum of activities required to influence favourably the underlying cause of cardiovascular disease, as well as to provide the best possible physical, mental and social conditions, so that the patients may, by their own efforts, preserve or resume optimal functioning in their community and through improved health behaviour, slow or reverse progression of disease" (BACPR 2017, p.1). Typical components of CR consist of assessment and management plans, health behaviour change and education, lifestyle risk factor management (diet, smoking, exercise), medication management and psychosocial health (Dalal 2015; Thomas 2019). Psychosocial components in CR programmes were introduced to enhance $\mathrm{HRQ}$ oL, increase treatment adherence and improve cardiovascular prognosis (Pogosova 2015). However, often dependent on resource availability and geographic location, CR programmes vary in their core components, intensity, duration, setting and delivery team. For example, less than one quarter of CR programmes across the UK provide specific psychological treatments (NACR 2018). Furthermore, a recent cross-sectional study of CR in 111 countries identified significant regional variation and relatively low numbers of programmes that included a psychosocial component (Supervia 2019). Notwithstanding, the benefits of CR are well established, including increased HRQoL, and reductions in rehospitalisation, morbidity and mortality rates (Anderson 2016; Long 2019). This review will include psychological interventions delivered alone or with CR, as long as CR was part of usual medical care and offered routinely to both trial arms.

Evidence-based guidelines recommend medication (e.g. selective serotonin reuptake inhibitors or selective serotonin norepinephrine reuptake inhibitors) as a first-step therapy in the treatment of major depressive disorder (MDD; Jobst 2016; Qaseem 2016). Current evidence reports that adjunct use of pharmacotherapy for an underlying psychological condition may increase intervention effectiveness compared with interventions without

Psychological interventions for depression and anxiety in patients with coronary heart disease, heart failure or atrial fibrillation 
pharmacotherapy (Richards 2017; Richards 2018). However, pharmacotherapy treatment for mental health disorders can present cardiovascular risk due to side effects of the drugs or interactions with other medications (Pina 2018). People most likely to receive medication are those with the most severe forms of depressive illness (Gartlehner 2017). Including people across the spectrum of disease severity is essential to determining who benefits the most from psychological intervention. This review will include psychological interventions, with and without adjunct pharmacotherapy.

\section{How the intervention might work}

There is considerable evidence that depression and anxiety are risk factors for the development of HD (Dhar 2016), with depression an established independent risk factor of CHD (Van Der Kooy 2007). Both are strongly associated with poor cardiac outcomes (Carney 2017; May 2017; Seldenrijk 2015). Those with established HD are likely to experience depression and anxiety with prevalence rates frequently reported at around $40 \%$ (Colquhoun 2013; Seldenrijk 2015), and some large-scale trials reporting levels of depression over 60\% (Carney 2004; Kotseva 2009). Hence, the evidence for a bidirectional relationship between negative emotional states and HD is substantial. While the pathways are not completely understood, factors impacting on poorer prognosis of people with HD and with depression or anxiety, or both, are multifactorial, including lifestyle, behavioural and, more recently, biological. Lifestyle and behavioural factors include increased rates of smoking, alcohol intake, physical inactivity, and obesity (Dhar 2016). MDD is also a predictor of poor disease treatment, management and medication adherence (Bauer 2012; Goldstein 2017). For example, patients who are depressed are far less likely to be motivated to complete or even attend CR programmes (Chauvet-Gelinier 2017). MDD can cause autonomic nervous system dysfunction, elevated cortisol levels, and elevated markers of inflammation with subsequent deleterious downstream effects, including hypertension, left ventricular hypertrophy, and coronary vasoconstriction (Dhar 2016). The evidence is building to suggest that both depression and anxiety contribute to the pathogenesis of HD (O'Neill 2016; Seldenrijk 2015). Of significance is the growing recognition of the role of psychological determinants in cardiac illness and recovery (Chauvet-Gelinier 2017), which has implications for psychological interventions and subsequent adoption and maintenance of healthy lifestyle behaviours, medication adherence and potential for improved clinical outcomes in those with HD.

\section{Why it is important to do this review}

In their 2017 Cochrane Review, Richards 2017, according to GRADE methodology, concluded that uncertainty remains regarding the benefits of psychological interventions among people with CHD (i.e. reducing cardiac mortality and reducing psychological symptoms), and large-scale trials are still warranted. The current review will include $\mathrm{HF}$ and $\mathrm{AF}$ in addition to $\mathrm{CHD}$, thus increasing the likelihood of the inclusion of additional large-scale trials in the existing evidence base. Adding to the uncertainty was the number of trials with no reported psychopathologies at baseline (Richards 2017). This review will include people with HD with reported levels (including scores indicating absence) of depression or anxiety at baseline, which will add to the knowledge base on the effectiveness of optimally targeted psychological interventions. Further, many national and international CVD secondary prevention and rehabilitation guidelines acknowledge the importance of the provision of psychological interventions in addition to standard rehabilitation (Lichtman 2014; NICE 2009; Piepoli 2016; Woodruffe 2015), especially in people with depression or anxiety with comorbid CVD, and will therefore benefit from the evidence of this review.

\section{O B JECT IVES}

To assess the efficacy and costs of psychological interventions (alone, or with CR or pharmacotherapy, or both) in adults who have a diagnosis of $\mathrm{CHD}, \mathrm{HF}$ or $\mathrm{AF}$ compared to no intervention, or treatment as usual, on psychological and clinical outcomes.

\section{METHODS}

\section{Criteria for considering studies for this review}

\section{Types of studies}

Randomised controlled trials (RCTs) with parallel-group, individual participant or cluster-allocation design comparing the independent effects of a psychological intervention versus a usualcare comparator. We will consider RCTs where follow-up was six months or more following the start of the intervention or randomisation. We will include trials reported as full text, those published as abstract only, and unpublished data.

\section{Types of participants}

Adults, 18 years of age and older with HD, with and without depression or anxiety, managed in either hospital or community settings. Participants with HD will include people who had a clinical diagnosis of CHD, HF or AF. Participants with CHD will include those who have experienced a myocardial infarction (MI), a revascularisation procedure, such as coronary artery bypass graft (CABG) or percutaneous coronary intervention $(\mathrm{PCl})$, and people with angina, or angiographically defined CHD. Participants with HF will include reduced $(<40 \%)$ ejection fraction (HFrEF), mid-range ( $40 \%$ to $49 \%$ ) ejection fraction (HFmrEF) and those with preserved ( $\geq 50 \%$ ) ejection fraction HF (HFpEF; Ponikowski 2016; Watson 2018). We will include participants with all types of AF. Participants may have comorbid conditions (alongside HD).

However, we will exclude trials if the population is mixed, for example, participants with CHD or diabetes, and the data are not stratified by condition. Only if more than $50 \%$ of participants are ineligible for this review will we exclude the trial. We will also exclude trials including participants without a baseline assessment of depression or anxiety, patient-reported or otherwise. Prior to excluding any such trials, we will contact the trial authors to request the data for the subgroup of interest.

\section{Types of interventions}

We will consider all psychological interventions targeting depression or anxiety delivered by psychologists or healthcare workers with specific training in psychological techniques. Included interventions may vary in content and composition, ranging from cognitive behavioural therapies (e.g. rational emotive therapy, restructuring), to third-wave cognitive behavioural therapies (e.g. mindfulness-based stress reduction). We will classify psychological interventions as per the Cochrane Common Mental Disorders (CCMD) classification of psychological interventions (CCMD 2019). We will exclude less specific approaches, such 
as therapeutic counselling or educational interventions from this delineation, along with self-management techniques used to change cardiac risk factors such as smoking and low levels of exercise. We will exclude trials that do not specify that healthcare workers delivering the intervention were specifically trained, or following a treatment protocol. When uncertainty exists, prior to excluding any such trials, we will contact the trial authors to determine whether specific training took place. We will exclude trials where evaluation of the intervention is solely directed at improving adherence to other cardiovascular efficacious treatments (e.g. medications, exercise) or modifying lifestyle factors (e.g. smoking, diet). We will only consider trials where the effect of the psychological intervention can be evaluated independently.

We will include trials that compare psychological treatment with usual care. Usual care may include routine medical care provided to people with $\mathrm{HD}$, and co-interventions including referral to or participation in (or both) a comprehensive CR programme. Although psychological interventions often include co-interventions (e.g. cardiac risk factor education), we will exclude trials where the co-interventions are not offered in usual care. We will include trials of psychological interventions offered in conjunction with pharmacotherapy (Richards 2017). These interventions will be eligible for inclusion as long as the effect of the psychological intervention can be evaluated independently from co-interventions.

\section{Types of outcome measures}

\section{Primary outcomes}

1. Depression (continuous outcome)

2. Depression (dichotomous outcome)

3. Anxiety (continuous outcome)

4. Anxiety (dichotomous outcome)

We will measure depression and anxiety as change in symptoms (mean score), unless trials report binary data (cut-off points) only. We will analyse continuous and dichotomous outcomes separately and we will accept psychometrically validated measures of depression or anxiety, self-reported or other, along with clinical diagnosis. The primary time point for anxiety and depression will be at six-month follow-up, and similarly for secondary outcomes.

Validated tools for depression include: Beck's Depression Inventory (BDI; Beck 1961); Beck's Depression Inventory II (BDI II; Beck 1996); Behavioral Activation for Depression Scale (BADS; Kanter 2007); Cardiac Depression Scale (CDS; Hare 1996); Center for Epidemiological Studies Depression Scale (CES-D; Radloff 1977); Comprehensive Psychopathological Rating Scale (CPRS; Asberg 1978); Comprehensive Psychopathological Rating Scale SelfAffective (CPRS S-A; Svanborg 1994); Depression, Anxiety and Stress Scale (DASS21; Lovibond 1995); Delusions-Symptoms-States Inventory/states of Anxiety and Depression (DSSI/SAD; Bedford 1976); Hospital Anxiety and Depression Scales (HADS-D; Zigmond 1983); Hamilton Depression Rating Scale (HAM-D; Hamilton 1960); Maastricht Questionnaire for Vital Exhaustion and Depression (Williams 2010); Montgomery-Åsberg Depression Rating Scale (MADRS; Montgomery 1979); Patient Health Questionnaire-9 (PHQ-9; Kroenke 2001); Symptom Checklist 90-R (SCL-90-R; Derogatis 1992); Zung Self-Rating Depression Scale (ZDS; Zung 1965).
Validated tools for anxiety include: Beck's Anxiety Inventory (BAl; Beck 1988); Cardiac Anxiety Questionnaire (CAQ; Eifert 2000); Delusions Symptoms States Inventory/states of Anxiety and Depression (DSSI/SAD; Bedford 1976); Generalised Anxiety Disorder Assessment (GAD-7; Spitzer 2006); Hospital Anxiety and Depression Scales (HADS-A; Zigmond 1983); Overall Anxiety Severity and Impairment Scale 9OASIS (Campbell-Sills 2009); StateTrait Anxiety Inventory (STAI; Spielberger 1983); Symptom Checklist 90-R (SCL-90-R; Derogatis 1992); Taylor Manifest Anxiety Scale (Taylor 1953).

\section{Secondary outcomes}

1. HRQoL

2. Self-efficacy

3. All-cause mortality

4. Cardiovascular mortality

5. All-cause hospitalisations (participants with at least one event)

6. Cardiovascular hospitalisations (participants with at least one event)

7. Cardiovascular morbidity: non-fatal MI (participants with at least one event)

8. Cardiovascular morbidity: revascularisation (CABG, PCI; participants with at least one event), disability-adjusted life year (DALYs), costs

9. Intervention acceptability

10. Return to work

11.Costs and cost-effectiveness: costs per quality-adjusted life years (QALYs)

12.Adverse events

Validated tools for HRQoL include: Cantril Self-Anchoring Striving Scale (Ladder of Life; Cantril 1965); Chronic Heart Failure Assessment Tool (CHAT; Dunderdale 2008); Chronic Heart Failure Questionnaire (CHFQ; Guyatt 1989); Dartmouth COOP scales (Nelson 1987); EuroQol-5D (EQ-5D; Rabin 2001); HeartQoL (Oldridge 2014); Kansas City Cardiomyopathy Questionnaire (KCCQ; Green 2000); Karnofsky Performance Status Scale (Schag 1984); Left Ventricular Disease Questionnaire (LVDQ; O'Leary 2000); MacNew Questionnaire (Valenti 1996); Minnesota Living with Heart Failure Questionnaire (LHFQ; Rector 1987); Myocardial Infarction Dimensional Assessment Scale (MIDAS; Thompson 1998); Psychological General Well-Being Index (Dupuy 1984); Quality of Life Questionnaire in Severe Heart Failure (QLQ-SHF; Wiklund 1987); Seattle Angina Questionnaire (Spertus 1995); Short Form Health Survey 36 (SF-36; Ware 1994); Short Form Health Survey (SF-12; Ware 1995).

Validated tools for self-efficacy include: Generalized Self-Efficacy Scale (GES; Schwarzer 1995); General Self-Efficacy Scale (GES-6; Romppel 2013); New General Self-Efficacy Scale (NGSE; Chen 2001); Self-efficacy Survey (Panc 2013).

We will assess intervention acceptability using 1 ) retention rates and 2) reporting a narrative summary of qualitative data on utility, adherence, barriers and satisfaction (Sekhon 2017; Saracutu 2018).

We will report adverse events in a narrative summary, including a notable absence of evidence addressing whether there are adverse outcomes arising from participation in a psychological intervention.

Psychological interventions for depression and anxiety in patients with coronary heart disease, heart failure or atrial fibrillation 
Reporting one or more of the above-listed primary and secondary outcomes in the trial is not an inclusion criterion for the review. Where a published report does not appear to report one of these outcomes, we will access the trial protocol and contact the trial authors to ascertain whether the outcomes were measured but not reported.

Where reported we will assess primary and secondary outcomes at time points longer than 12 months and capture the longest available follow-up. As part of the narrative, we will include in the review relevant trials, which measured these outcomes but did not report the data at all, or not in a usable format.

\section{Search methods for identification of studies}

\section{Electronic searches}

We will identify trials through systematic searches of the following bibliographic databases:

1. Cochrane Central Register of Controlled Trials (CENTRAL) in the Cochrane Library

2. MEDLINE (Ovid, from 2009 onwards)

3. Embase (Ovid, from 2009 onwards)

4. PsycINFO (Ovid, from 2009 onwards)

5. CINAHL (EBSCO, from 2009 onwards)

We will adapt the preliminary search strategy (see (Appendix 1) for MEDLINE (Ovid) for use in the other databases and we will apply the Cochrane Sensitivity-precision maximising RCT filter to MEDLINE (Ovid) and adaptations of it to the other databases, except CENTRAL (Lefebvre 2019).

We will also conduct a search of ClinicalTrials.gov (www.ClinicalTrials.gov), and the World Health Organization International Clinical Trials Registry Platform (WHO ICTRP) Search Portal (apps.who.int/trialsearch/), for ongoing or unpublished trials.

We will search all databases from 2009 (in accordance with NICE 2009 guidelines) to the present, and we will impose no restriction on language of publication or publication status. In 2009 there were many scientific advances in understanding the relationship between depression and CVD, with lasting implications for the treatment of depression in people with CVD (Davidson 2010). These advances included publication of results from three RCTs reporting the efficacy of depression interventions in people with CVD (Carney 2009; Freedland 2009; Rollman 2009). In late 2008 the American Heart Association released a science advisory, Depression and coronary heart disease: recommendations for screening, screening referral and treatment (Lichtman 2014). In 2009 NICE first published guidelines for effective delivery of psychological interventions for depression among those with chronic health conditions, with particular attention paid to heart disease (NICE 2009). The guidelines advocated a more structured approach to guide interventions and stipulated that interventions of this type be delivered by competent delivery agents or practitioners (NICE 2009).

We will not perform a separate search for adverse effects resulting from psychological interventions used for the treatment of depression or anxiety. We will consider adverse effects described in included trials only.

\section{Searching other resources}

We will search by hand the reference lists of all included trials and previous systematic reviews for additional references to trials. We will contact the main authors of included trials and experts within the area to ask for any missed, unreported or ongoing trials. We will also search for any retraction statements and errata for included trials.

\section{Data collection and analysis}

\section{Selection of studies}

Two review authors (CFS and KMcG) will independently screen titles and abstracts for inclusion of all the potential trials we identify as a result of the search and code them as 'retrieve' (eligible or potentially eligible/unclear) or 'do not retrieve'. If there are any disagreements, a third author will be asked to arbitrate (JDL). We will retrieve the full-text trial reports/publication and two review authors (CFS and KMcG) will independently screen the full-text and identify trials for inclusion, and identify and record reasons for exclusion of the ineligible trials. We will resolve any disagreement through discussion or, if required, we will consult a third person (JDL). We will identify and exclude duplicates and collate multiple reports of the same trial so that each trial rather than each report is the unit of interest in the review. We will record the selection process in sufficient detail to complete a PRISMA flow diagram and 'Characteristics of excluded studies' table (Liberati 2009).

\section{Data extraction and management}

We will use a data collection form for trial characteristics and outcome data, which has been piloted on at least one trial in the review (Richards 2017). One review author (CFS and KMcG) will extract trial characteristics from included trials. We will extract the following study characteristics.

1. Methods: trial design, total duration of trial, number of trial centres and location, trial setting, and date of trial

2. Participants: number randomised, number lost to follow-up/ withdrawn, number analysed, mean age, age range, gender, severity of condition, diagnostic criteria, inclusion criteria, and exclusion criteria

3. Interventions: intervention (including the goals of treatment and components used to achieve those goals), comparison, and co-interventions. We will describe psychological interventions as per CCMD classifications.

4. Outcomes: primary and secondary outcomes specified and collected, and time points reported

5. Notes: funding for trial, and notable conflicts of interest of trial authors

Two review authors (CFS and KMCG) will independently extract outcome data from included trials. We will resolve disagreements by consensus or by involving a third person (JDL). However, we will extract only 'new' variables for papers that were included in Richards 2017. One review author (CFS) will transfer data into the Review Manager 5 (Review Manager 2014) file. We will doublecheck that data are entered correctly by comparing the data presented in the systematic review with the data extraction form. A second review author (KMCG) will spot-check trial characteristics for accuracy against the trial report. 


\section{Assessment of risk of bias in included studies}

Two review authors (CFS and KMCG) will independently assess risk of bias for each trial according to the Cochrane 'Risk of bias' tool, RoB2 (Higgins 2019a). We will resolve any disagreements by discussion or by involving another review author (JDL). We will assess the risk of bias of specific results of a trial according to the following domains:

1. Bias arising from the randomisation process

2. Bias due to deviations from intended interventions

3. Bias due to missing outcome data

4. Bias in measurement of the outcome

5. Bias in selection of the reported result

6. Bias arising from identification or recruitment of individual participants within clusters

We included domain 6 as per the revised Cochrane 'Risk of bias tool' for randomized trials (RoB 2) (Sterne 2019). If cluster-RCTs are included we will consider whether the reported data analysis had appropriately taken account of the aggregate nature of the data. We will pay particular attention to randomisation processes (e.g. allocation sequence, timing, baseline imbalances) and blinding (e.g. recruitment bias, identification bias) (Eldridge 2016).

We will assess the risk of bias for the outcomes of the included trials that will be included in our 'Summary of findings' table according to the effect of assignment to the interventions at baseline, regardless of whether the interventions are received as intended (the 'intention-to-treat effect').

We will use the signalling questions in RoB2 and rate each domain as 'low risk of bias', 'some concerns' or 'high risk of bias'. We will summarise the 'Risk of bias' judgements across different trials for each of the domains listed for each outcome. The overall risk of bias for the result will be the least favourable assessment across the domains of bias. Where information on risk of bias relates to unpublished data or correspondence with a trial author, we will note this in the 'Risk of bias' table. Thus, "A judgement of 'High' risk of bias for any individual domain will lead to the result being at 'High' risk of bias overall, and a judgement of 'Some concerns' for any individual domain will lead to the result being at 'Some concerns', or 'High' risk, overall" (Sterne 2019).

When considering treatment effects, we will take into account the risk of bias for the trials that contribute to that outcome.

\section{Assessment of bias in conducting the systematic review}

We will conduct the review according to this published protocol and report any deviations from it in the 'Differences between protocol and review' section of the systematic review.

\section{Measures of treatment effect}

Dichotomous outcomes for each comparison will be expressed as risk ratios with $95 \%$ confidence intervals. Continuous data will be expressed as mean difference with $95 \%$ confidence intervals, or where an outcome is measured and reported in more than one way, as standardised mean difference with 95\% confidence intervals. We will express continuous data as the mean change (and standard deviation (SD)) in outcomes between baseline and followup for both psychological intervention and control groups; when not available, we will use the absolute mean (and SD) outcome at follow-up for both groups. We will report event trial sample sizes based on the number randomised to treatment conditions.

We will consider treatment effects for depression, anxiety and HRQoL in terms of clinically meaningful differences, for example, we will consider a 1.5 difference on the HADS as clinically meaningful (Puhan 2008), and a 5-point difference on the MLWHF questionnaire as clinically meaningful (Rector 1992).

We will narratively describe skewed data reported as medians and interquartile ranges.

\section{Unit of analysis issues}

For trials with more than one relevant intervention arm included in the same analysis, we will divide the number randomised in the control group by the number of intervention arms to obtain the denominator for data analysis. In accordance with Section 9.3.4 of the Cochrane Handbook for Systematic Reviews of Interventions (Higgins 2019b), if we include data with repeated observations on participants, we will define several outcomes based on different periods of follow-up and conduct separate analyses. Analysis will include all trials with measurement at the end of intervention (post-test). If we include cluster-RCTs, we will consider whether the reported data analysis has appropriately taken account of the aggregate nature of the data.

\section{Dealing with missing data}

We will contact investigators or trial sponsors in order to verify key trial characteristics and obtain missing numerical outcome data where possible (e.g. when a trial is identified as abstract only). Where possible, we will use the Review Manager 5 calculator to calculate missing SDs using other data from the trial, such as confidence intervals (Review Manager 2014). Where this is not possible, and the missing data are thought to introduce serious bias, we will explore the impact of including such trials in the overall assessment of results by a sensitivity analysis.

\section{Assessment of heterogeneity}

We will inspect forest plots visually to consider the direction and magnitude of effects and the degree of overlap between confidence intervals. We will use the $I^{2}$ statistic (Higgins 2003), to measure heterogeneity among the trials in each analysis, but acknowledge that there is substantial uncertainty in the value of $\mathrm{I}^{2}$ statistic when there is only a small number of trials. We will also consider the $\mathrm{P}$ value from the $\mathrm{Chi}^{2}$ test (Deeks 2019). If we identify substantial heterogeneity we will report it and explore possible causes by prespecified subgroup analysis.

We will use a threshold of $\mathrm{I}^{2}$ statistic value greater than $50 \%$ (considered to represent substantial heterogeneity; section 10.10 .2 of the Cochrane Handbook for Systematic Reviews of Interventions) for both dichotomous and continuous outcomes to determine the statistical model to be used for meta-analysis (Deeks 2019).

\section{Assessment of reporting biases}

If we are able to pool more than 10 trials, we will create and examine a funnel plot and the Egger test to explore possible small trial biases for the primary outcomes (Egger 1997).

Psychological interventions for depression and anxiety in patients with coronary heart disease, heart failure or atrial fibrillation 


\section{Data synthesis}

We will undertake meta-analyses only where this is meaningful, that is, if the treatments, participants and the underlying clinical question are similar enough for pooling to make sense. As heterogeneity is expected for psychological interventions, we will pool data from each trial using a random-effects model. This will provide a more conservative statistical comparison of the difference between intervention and control because a confidence interval around the effect estimate is wider than a confidence interval around a fixed-effect estimate. Due to the tendency of smaller trials, which are more susceptible to publication bias, to be over-weighted with a random-effects model we will also conduct a sensitivity analysis using the fixed-effect pooled estimate and $95 \%$ confidence interval (Heran 2008a; Heran 2008b). We will complete data synthesis and analyses using Review Manager 5 software (Review Manager 2014). For primary and secondary outcomes, where there were insufficient data, or where it is inappropriate to combine trials statistically, we will present a narrative review using the vote-counting method (Hedges 1980).

\section{'Summary of findings' table}

We will create a 'Summary of findings' table using the following outcomes: depression, anxiety, HRQoL, all-cause mortality, cardiovascular mortality, cardiovascular hospitalisations and costeffectiveness. We will use the five GRADE considerations (trial limitations, consistency of effect, imprecision, indirectness and publication bias) to assess the certainty of a body of evidence as it relates to the trials that contribute data to the metaanalyses for the prespecified outcomes. We will use methods and recommendations described in Chapter 12 of the Cochrane Handbook for Systematic Reviews of Interventions (Schünemann 2019), using GRADEpro software (GRADEpro GDT). We will justify all decisions to downgrade the certainty of trials using footnotes and we will make comments to aid reader's understanding of the review where necessary.

Two review authors (CFS and KMCG), working independently, will make Judgements about evidence certainty with disagreements resolved by discussion or by involving a third review author (JDL). We will justify judgements, document them and incorporate them into reporting of results for each outcome.

We plan to extract trial data, format our comparisons in data tables and prepare a 'Summary of findings' table before writing the results and conclusions of our review. A template 'Summary of findings table' is included as Table 1.

\section{Subgroup analysis and investigation of heterogeneity}

We plan to carry out the following subgroup analyses.

1. Trials of psychological interventions with and without cointervention of CR

2. Trials of psychological interventions with and without cointervention of pharmacotherapy

3. Trials of psychological interventions with and without cointerventions of $\mathrm{CR}$ and pharmacotherapy
4. Trials of psychological interventions targeting HD population (CHD or $\mathrm{AF}$ or $\mathrm{HF}$ )

5. Trials of psychological interventions targeting population with depression or anxiety, or those not specified

6. Trials with analysis of mode of psychological intervention (individual or group or combined individual and group)

7. Trials of psychological interventions with and without family involved in intervention

8. Trials of psychological interventions specifically targeting depression or anxiety or not

9. Trials that employ different treatment components, that is, relaxation, stress management techniques, cognitive techniques, emotional support or client-led discussion, or combinations

We will explore potential heterogeneity in psychological interventions via three approaches:

1. detailed tabulation of population, intervention and comparators for each trial;

2. within-trial subgroup analyses (supported by subgroup $x$ intervention/control interaction terms); and

3. between-trial analyses via meta-regression.

We will use meta-regression to examine the association between effects of all outcomes this applies to that are reported up to six months. We will perform meta-regression analyses using the 'metareg' command in STATA (StataCorp 2019).

\section{Sensitivity analysis}

We plan to carry out the following sensitivity analyses, to test whether key methodological factors or decisions have affected the main result.

1. Only including trials with a low risk of bias according to the RoB2 tool

2. When it is not possible to calculate missing SDs and the missing data are thought to introduce bias

3. The assumption of heterogeneity using a fixed-effect pooled estimate and $95 \%$ confidence interval

\section{Reaching conclusions}

We will base our conclusions only on findings from the quantitative or narrative synthesis of included trials for this review. We will avoid making recommendations for practice and our implications for research will suggest priorities for future research and outline what the remaining uncertainties are in the area.

\section{ACKNOWLEDGEMENTS}

The authors acknowledge the provision of a template protocol by Cochrane Heart, the support given by the peer/consumer reviewers of the Cochrane Heart editorial team and the contact editor Dr Bill Cayley and peer reviewers Dr Jalila Jbilou and Dr Walid Kamal Abdelbasset. 


\section{REFERE N CE S}

\section{Additional references}

\section{Anderson 2014}

Anderson L, Taylor RS. Cardiac rehabilitation for people with heart disease: an overview of Cochrane systematic reviews. Cochrane Database of Systematic Reviews 2014, Issue 12. [DOI: 10.1002/14651858.CD011273.pub2]

\section{Anderson 2016}

Anderson L, Oldridge N, Thompson DR, Zwisler AD, Rees K, Martin N, et al. Exercise-based cardiac rehabilitation for coronary heart disease: Cochrane systematic review. Journal of the American College of Cardiology 2016;67:1-12.

\section{Asberg 1978}

Asberg M, Montgomery SA, Perris C, Schalling D, Sedvall G. A comprehensive psychopathological rating scale. Acta Psychiatrica Scandinavica 1978;271:5-27.

\section{BACPR 2017}

British Association for Cardiovascular Prevention and Rehabilitation. The BACPR Standards and Core Components for Cardiovascular Disease Prevention and Rehabilitation 2017. 3rd Edition. London: British Cardiovascular Society, 2017.

\section{Bauer 2012}

Bauer LK, Caro MA, Beach SR, Mastromauro CA, Lenihan E, Januzzi JL, et al. Effects of depression and anxiety improvement on adherence to medication and health behaviors in recently hospitalized cardiac patients. American Journal of Cardiology 2012;109:1266-71.

\section{Beck 1961}

Beck AT, Ward CH, Mendelson M, Mock J, Erbaugh J. An inventory for measuring depression. Archives of General Psychiatry 1961;4:561-71.

\section{Beck 1988}

Beck AT, Epstein N, Brown G, Steer RA. An inventory for measuring clinical anxiety: psychometric properties. Journal of Consulting and Clinical Psychology 1988;56:893-7.

\section{Beck 1996}

Beck AT, Steer RA, Brown GK. Manual for the Beck Depression Inventory-II. San Antonio, TX. Psychological Corporation, 1996.

\section{Bedford 1976}

Bedford A, Foulds GA, Sheffield BF. A New Personal Disturbance Scale (DSSI/SAD). British Journal of Social and Clinical Psychology 1976;15:387-94.

\section{Benjamin 2017}

Benjamin EJ, Blaha MJ, Chiuve SE, Cushman M, Das SR, Deo R, et al. Heart disease and stroke statistics-2017 update: a report from the American Heart Association. Circulation 2017;135:e146-e603.

\section{Bosworth 2018}

Bosworth HB, Blalock DV, Hoyle RH, Czajkowski SM, Voils Cl. The role of psychological science in efforts to improve cardiovascular medication adherence. American Psychologist 2018;73:968-80.

\section{Campbell-Sills 2009}

Campbell-Sills L, Norman SB, Craske MG, Sullivan G, Lang AJ, Chavira DA, et al. Validation of a brief measure of anxietyrelated severity and impairment: the Overall Anxiety Severity and Impairment Scale (OASIS). Journal of Affective Disorders 2009;112:92-101.

\section{Cantril 1965}

Cantril H. The pattern of Human Concerns. New Brunswick, NJ: Rutgers University Press, 1965.

\section{Carney 2004}

Carney RM, Blumenthal JA, Freedland KE, Youngblood M, Veith RC, Burg MM, et al. Depression and late mortality after myocardial infarction in the Enhancing Recovery in Coronary Heart Disease (ENRICHD) study. Psychosomatic Medicine 2004;66:466-74.

\section{Carney 2009}

Carney RM, Freedland KE, Rubin EH, Rich MW, Steinmeyer BC Harris WS. Omega-3 augmentation of sertraline in treatment of depression in patients with coronary heart disease: a randomized controlled trial. Journal of the American Medical Association 2009;302:1651-7.

\section{Carney 2017}

Carney RM, Freedland KE. Depression and coronary heart disease. Nature Reviews Cardiology 2017;14:145-55.

\section{CCMD 2019}

Cochrane Common Mental Disorders. Psychological therapies topics list. Available at cmd.cochrane.org/scope-our-work (accessed 17 December 2019).

\section{Chauvet-Gelinier 2017}

Chauvet-Gelinier JC, Bonin B. Stress, anxiety and depression in heart disease patients: a major challenge for cardiac rehabilitation. Annals of Physical Rehabilitation Medicine 2017;60:6-12.

\section{Chen 2001}

Chen G, Gully SM, Eden D. Validation of a new general selfefficacy scale. Organizational Research Methods 2001;4:62-83.

\section{Colquhoun 2013}

Colquhoun DM, Bunker SJ, Clarke DM, Glozier N, Hare DL, Hickie IB, et al. Screening, referral and treatment for depression in patients with coronary heart disease. Medical Journal of Australia 2013;198:483-4.

\section{Cozzolino 2018}

Cozzolino P, Ambrosio G, Bassand J-P, Cate HT, Cools F, Darious $\mathrm{H}$, et al. The economic burden attributable to atrial 
fibrillation in nine European countries: perspectives from the GARFIELD-AF registry. European Heart Journal 2018;39(Suppl 1):P2889.

\section{Dalal 2015}

Dalal HM, Doherty P, Taylor RS. Cardiac rehabilitation. BMJ 2015;351:h5000.

\section{Davidson 2010}

Davidson KW, Korin MR. Depression and cardiovascular disease: selected findings, controversies, and clinical implications from 2009. Cleveland Clinic Journal of Medicine 2010;77:S20-6.

\section{Deeks 2019}

Deeks JJ, Higgins JP, Altman DG (editors). Chapter 10: Analysing data and undertaking meta-analyses. In: Higgins JP, Thomas J, Chandler J, Cumpston M, Li T, Page MJ, Welch VA (editors). Cochrane Handbook for Systematic Reviews of Interventions version 6.0 (updated July 2019). Cochrane, 2019. Available from www.training.cochrane.org/handbook.

\section{Derogatis 1992}

Derogatis LR. SCL-90-R, Administration, Scoring and Procedures Manual-II for the R(evised) Version and Other Instruments of the Psychopathology Rating Scale Series. Townson: Clinical Psychometric Research, 1992.

\section{Dhar 2016}

Dhar AK, Barton DA. Depression and the link with cardiovascular disease. Frontiers in Psychiatry / Frontiers Research Foundation 2016;7:33.

\section{Dunderdale 2008}

Dunderdale K, Thompson DR, Beer SF, Furze G, Miles JN. Development and validation of a patient-centered healthrelated quality-of-life measure: the chronic heart failure assessment tool. Journal of Cardiovascular Nursing 2008;23:364-70.

\section{Dupuy 1984}

Dupuy $\mathrm{H}$. The Psychological General Well-Being (PGWB) Index. In: NK Wenger, ME Mattson, CD Furberg, J Elinson, Eds. Assessment of quality of life in clinical trials of cardiovascular therapies. Greenwich, Conn: Le Jacq Publishing, Inc, 1984.

\section{Egger 1997}

Egger M, Smith GD, Schneider M, Minder C. Bias in meta-analysis detected by a simple, graphical test. BMJ 1997;315:629.

\section{Eifert 2000}

Eifert GH, Thompson RN, Zvolensky MJ, Edwards K, Frazer NL, Haddad JW, et al. The cardiac anxiety questionnaire: Development and preliminary validity. Behaviour Research and Therapy 2000;38:1039-53.

\section{Eldridge 2016}

Eldridge S, Campbell M, Campbell M, Dahota A, Giraudeau B, Higgins J, et al. Revised Cochrane risk of bias tool for randomized trials (RoB 2.0): additional considerations for cluster-randomized trials. (acessed 20 October 2019). Creative Commons Attribution-NonCommercialNoDerivatives 4.0
International License, 2016 www.bristol.ac.uk/media-library/ sites/social-community-medicine/images/centres/cresyda/ RoB2-0_cluster_parallel_guidance.pdf.

\section{Freedland 2009}

Freedland KE, Skala JA, Carney RM, Rubin EH, Lustman PJ, Dávila-Román VG, et al. Treatment of depression after coronary artery bypass surgery: a randomized controlled trial. Archives of General Psychiatry 2009;66:387-96.

\section{Freedland 2015}

Freedland KE, Carney RM, Rich MW, Steinmeyer BC, Rubin EH. Cognitive behavior therapy for depression and self-care in heart failure patients: a randomized clinical trial. JAMA Internal Medicine 2015;175:1773-82.

\section{Gale 2014}

Gale CR, Batty GD, Osborn DP, Tynelius P, Rasmussen F. Mental disorders across the adult life course and future coronary heart disease: evidence for general susceptibility. Circulation 2014;129:186-93.

\section{Garster 2009}

Garster NC, Palta M, Sweitzer NK, Kaplan RM, Fryback DG. Measuring health-related quality of life in population-based studies of coronary heart disease: comparing six generic indexes and a disease-specific proxy score. Quality of Life Research 2009;18:1239-47.

\section{Gartlehner 2017}

Gartlehner G, Wagner G, Matyas N, Titscher V, Greimel J, Lux L, et al. Pharmacological and non-pharmacological treatments for major depressive disorder: review of systematic reviews. BMJ Open 2017;7:e014912.

\section{Gheorghe 2018}

Gheorghe A, Griffiths U, Murphy A, Legido-Quigley H, Lamptey P, Perel P. The economic burden of cardiovascular disease and hypertension in low- and middle-income countries: a systematic review. BMC Public Health 2018;18:975.

\section{Giedrimiene 2017}

Giedrimiene D, King R. Burden of cardiovascular disease (CVD) on economic cost. Comparison of outcomes in US and Europe. Circulation. Cardiovascular Quality and Outcomes 2017;10:A207.

\section{Goldstein 2017}

Goldstein CM, Gathright EC, Garcia S. Relationship between depression and medication adherence in cardiovascular disease: the perfect challenge for the integrated care team. Patient Preference and Adherence 2017;11:547-59.

\section{GRADEpro GDT [Computer program]}

McMaster University (developed by Evidence Prime). GRADEpro GDT [GRADEpro Guideline Development Tool]. Version assessed 9 October 2019. Hamilton (ON): McMaster University (developed by Evidence Prime), 2015.

\section{Green 2000}

Green CP, Porter CB, Bresnahan DR, Spertus JA. Kansas City Cardiomyopathy Questionnaire: a new health status measure

Psychological interventions for depression and anxiety in patients with coronary heart disease, heart failure or atrial fibrillation 
for heart failure. Journal of the American College of Cardiology 2000;35:1245-55.

\section{Guyatt 1989}

Guyatt GH, Deyo RA, Charlson M, Levine MN, Mitchell A. Responsiveness and validity in health status measurement: a clarification. Journal of Clinical Epidemiology 1989;42:403-8.

\section{Hamilton 1960}

Hamilton M. A rating scale for depression. Journal of Neurology, Neurosurgery, and Psychiatry 1960;23:56-62.

\section{Hare 1996}

Hare DL, Davis CL. Cardiac depression scale: validation of a new depression scale for cardiac patients. Journal of Psychosomatic Research 1996;40:379-86.

\section{Hedges 1980}

Hedges LV, Olkin I. Vote-counting methods in research synthesis. Psychological Bulletin 1980;88:359-69.

\section{Heran 2008a}

Heran BS, Wong MM, Heran IK, Wright JM. Blood pressure lowering efficacy of angiotensin converting enzyme (ACE) inhibitors for primary hypertension. Cochrane Database of Systematic Reviews 2008, Issue 4. [DOI: 10.1002/14651858.CD003823]

\section{Heran 2008b}

Heran BS, Wong MM, Heran IK, Wright JM. Blood pressure lowering efficacy of angiotensin receptor blockers for primary hypertension. Cochrane Database of Systematic Reviews 2008, Issue 4. [DOI: 10.1002/14651858.CD003822]

\section{Higgins 2003}

Higgins JP, Thompson SG, Deeks JJ, Altman DG. Measuring inconsistency in meta-analyses. BMJ 2003;327:557-60.

\section{Higgins 2019a}

Higgins JP, Savović J, Page MJ, Elbers RG, Sterne JA. Chapter 8: Assessing risk of bias in a randomized trial. In: Higgins JP, Thomas J, Chandler J, Cumpston M, Li T, Page MJ, Welch VA (editors). Cochrane Handbook for Systematic Reviews of Interventions version 6.0 (updated July 2019). Cochrane, 2019. Available from www.training.cochrane.org/handbook.

\section{Higgins 2019b}

Higgins JP, Li T, Deeks JJ (editors). Chapter 6: Choosing effect measures and computing estimates of effect. In: Higgins JP, Thomas J, Chandler J, Cumpston M, Li T, Page MJ, Welch VA (editors). Cochrane Handbook for Systematic Reviews of Interventions version 6.0 (updated July 2019). Cochrane, 2019. Available from www.training.cochrane.org/handbook.

\section{Jobst 2016}

Jobst A, Brakemeier EL, Buchheim A, Caspar F, Cuijpers P, Ebmeier KP, et al. European Psychiatric Association guidance on psychotherapy in chronic depression across Europe. European Psychiatry 2016;33:18-36.

\section{Kanter 2007}

Kanter JW, Mulick PS, Busch AM, Berlin KS, Martell CR. The Behavioral Activation for Depression Scale (BADS): psychometric properties and factor structure. Journal of Psychopathology and Behavioral Assessment 2007;29:191-202.

\section{Kaplan 2009}

Sadock BJ, Sadock VA, Ruiz P. Kaplan and Sadock's Comprehensive Textbook of Psychiatry. 9th Edition. Philadelphia: Wolters Kluwer/Lippincott Williams \& Wilkins, 2009.

\section{Khanji 2018}

Khanj MY, Van Waardhuizen CN, Bicalho VV, Ferket BS, Hunink MG, Petersen SE. Lifestyle advice and interventions for cardiovascular risk reduction: a systematic review of guidelines. International Journal of Cardiology 2018;263:142-51.

\section{Kotseva 2009}

Kotseva K, Wood D, De Backer G, De Bacquer D, Pyörälä K, Keil U, et al. EUROASPIRE III: a survey on the lifestyle, risk factors and use of cardioprotective drug therapies in coronary patients from 22 European countries. European Journal of Cardiovascular Prevention and Rehabilitation 2009;16:121-37.

\section{Kroenke 2001}

Kroenke K, Spitzer RL, Williams JB. The PHQ-9: validity of a brief depression severity measure. Journal of General Internal Medicine 2001;16:606-13.

\section{Lefebvre 2019}

Lefebvre C, Glanville J, Briscoe S, Littlewood A, Marshall C, Metzendorf M-I, et al. Chapter 4: Searching for and selecting studies. In: Higgins JPT, Thomas J, Chandler J, Cumpston M, Li T, Page MJ, Welch VA (editors). Cochrane Handbook for Systematic Reviews of Interventions version 6.0 (updated July 2019). Cochrane, 2019. Available from www.training.cochrane.org/handbook.

\section{Lesyuk 2018}

Lesyuk W, Kriza C, Kolominsky-Rabas P. Cost-of-illness studies in heart failure: a systematic review 2004-2016. BMC Cardiovascular Disorders 2018;18:74.

\section{Liberati 2009}

Liberati A, Altman DG, Tetzlaff J, Mulrow C, Gotzsche PC, Ioannidis JP, et al. The PRISMA statement for reporting systematic reviews and meta-analyses of studies that evaluate health care interventions: explanation and elaboration. PLOS Medicine 2009;6(7):e1000100.

\section{Lichtman 2014}

Lichtman JH, Froelicher ES, Blumenthal JA, Carney RM, Doering LV, Frasure-Smith N, et al. Depression as a risk factor for poor prognosis among patients with acute coronary syndrome: systematic review and recommendations. Circulation 2014;129:1350-69.

\section{Long 2019}

Long L, Mordi IR, Bridges C, Sagar VA, Davies EJ, Coats AJ, et al. Exercise-based cardiac rehabilitation for adults with heart 
failure. Cochrane Database of Systematic Reviews 2019, Issue 1. [DOI: 10.1002/14651858.CD003331]

\section{Lovibond 1995}

Lovibond SH, Lovibond PF. Manual for the Depression Anxiety Stress Scales. Sydney: Psychology Foundation Australia, 1995.

\section{May 2017}

May HT, Horne BD, Knight S, Knowlton KU, Bair TL, Lappé DL, et al. The association of depression at any time to the risk of death following coronary artery disease diagnosis. European Heart Journal Quality of Care \& Clinical Outcomes 2017;3:296-302.

\section{Mensah 2017}

Mensah GA, Wei GS, Sorlie PD, Fine LJ, Rosenberg Y, Kaufmann PG, e al. Decline in cardiovascular mortality: possible causes and implications. Circulation Research 2017;120:366-80.

\section{Montgomery 1979}

Montgomery SA, Asberg M. A new depression scale designed to be sensitive to change. British Journal of Psychiatry 1979;134:382-89.

\section{NACR 2018}

British Heart Foundation. Quality and outcomes report 2018. The National Audit of Cardiac Rehabilitation. www.file:/// C:/Users/3052927/Downloads/nacr-report-2018_final.pdf (accessed 17 October 2019).

\section{Nelson 1987}

Nelson E, Wasson J, Kirk J, Keller A, Clark D, Dietrich A, et al. Assessment of function in routine clinical practice: description of the COOP chart method and preliminary findings. Journal of Chronic Diseases 1987;40 (Suppl 1):55S-63S.

\section{NICE 2009}

National Institute for Health and Clinical Excellence. Depression in adults with a chronic physical health problem: treatment and management. Clinical Guideline 91. London: National Institute for Health and Clinical Excellence, 2009.

\section{NICE 2018}

National Institute for Health and Clinical Excellence.

Cardiovascular disease risk assessment and prevention: treatment summary. bnf.nice.org.uk/treatment-summary/ cardiovascular-disease-risk-assessment-and-prevention.html accessed 15 April 2019.

\section{Oldridge 2014}

Oldridge, N, Höfer, S, McGee, H. The HeartQoL: part II. Validation of a new core health-related quality of life questionnaire for patients with ischemic heart disease. European Journal of Preventive Cardiology 2014;21:98-106.

\section{O'Leary 2000}

O'Leary CJ, Jones PW. The left ventricular dysfunction questionnaire (LVD-36): reliability, validity, and responsiveness. Heart 2000;83:634-40.

\section{O’Neill 2016}

O’Neill A, Fisher AJ, Kibbey KJ, Jacka FN, Kotowicz MA, Williams LJ, et al. Depression is a risk factor for incident coronary heart disease in women: an 18-year longitudinal study. Journal of Affective Disorders 2016;196:117-24.

\section{Panc 2013}

Panc T, Mihalcea A, Panc I. Self-Efficacy Survey: a new assessment tool. Procedia Social and Behavioural Sciences 2012;33:880-84.

\section{Piepoli 2016}

Piepoli MF, Hoes AW, Agewall S, Albus C, Brotons C, Catapano AL, et al. 2016 European Guidelines on cardiovascular disease prevention in clinical practice: the Sixth Joint Task Force of the European Society of Cardiology and Other Societies on Cardiovascular Disease Prevention in Clinical Practice (constituted by representatives of 10 societies and by invited experts): developed with the special contribution of the European Association for Cardiovascular Prevention \& Rehabilitation (EACPR). European Journal of Preventive Cardiology 2016;252:207-74.

\section{Pina 2018}

Piña IL, Di Palo KE, Ventura HO. Psychopharmacology and cardiovascular disease. Journal of the American College of Cardiology 2018;71:2346-59.

\section{Pogosova 2015}

Pogosova N, Saner H, Pedersen SS, Cupples ME, McGee H, Höfer S, et al. Psychosocial aspects in cardiac rehabilitation: from theory to practice. A position paper from the Cardiac Rehabilitation Section of the European Association of Cardiovascular Prevention and Rehabilitation of the European Society of Cardiology. European Journal of Preventive Cardiology 2015;10:1290-306.

\section{Ponikowski 2016}

Ponikowski P, Voors AA, Anker SD, Bueno H, Cleland JG, Coats AJ, et al. 2016 ESC Guidelines for the diagnosis and treatment of acute and chronic heart failure: the Task Force for the Diagnosis and Treatment of Acute and Chronic Heart Failure of the European Society of Cardiology (ESC) developed with the special contribution of the Heart Failure Association (HFA) of the ESC. European Heart Journal 2016;37:2129-200.

\section{Puhan 2008}

Puhan MA, Frey M, Büchi S, Schünemann HJ. The minimal important difference of the hospital anxiety and depression scale in patients with chronic obstructive pulmonary disease. Health Quality of Life Outcomes 2008;6:46.

\section{Qaseem 2016}

Qaseem A, Barry MJ, Kansagara D, Clinical Guidelines Committee of the American College of Physicians. Nonpharmacologic versus pharmacologic treatment of adult patients with major depressive disorder: a clinical practice guideline from the American College of Physicians. Annals of Internal Medicine 2016;164:350-9. 


\section{Rabin 2001}

Rabin R, de Charro. EQ-5D: a measure of health status from the EuroQol Group. Annals of Medicine 2001;33:337-43.

\section{Radloff 1977}

Radloff LS. The CES-D scale: a self-report depression scale for research in the general population. Applied Psychological Measurement 1977;1:385-401.

\section{Reavell 2018}

Reavell J, Hopkinson M, Clarkesmith D, Lane DA. Effectiveness of cognitive behavioral therapy for depression and anxiety in patients with cardiovascular disease: a systematic review and meta-analysis. Psychosomatic Medicine 2018;80:742-53.

\section{Rector 1987}

Rector TS, Kubo SH, Cohn JN. Patients' self-assessment of their congestive heart failure. Part 2: content, reliability and validity of a new measure, The Minnesota Living with Heart Failure Questionnaire. Heart Failure 1987;0ct/Nov:198-209.

\section{Rector 1992}

Rector TS, Cohn JN. Assessment of patient outcome with the Minnesota Living with Heart Failure questionnaire: reliability and validity during a randomized, double-blind, placebo controlled trial of pimobendan. Pimobendan Multicenter Research Group. American Heart Journal 1992;124:1017-25.

\section{Review Manager 2014 [Computer program]}

The Cochrane Collaboration. Review Manager 5 (RevMan 5). Version 5.3. Copenhagen: The Nordic Cochrane Centre: The Cochrane Collaboration, 2014.

\section{Richards 2017}

Richards SH, Anderson L, Jenkinson CE, Whalley B, Rees K, Davies $\mathrm{P}$, et al. Psychological interventions for coronary heart disease. Cochrane Database of Systematic Reviews 2017, Issue 4. [DOI: 10.1002/14651858.CD002902]

\section{Richards 2018}

Richards SH, Anderson L, Jenkinson CE, Whalley B, Rees K, Davies $\mathrm{P}$, et al. Psychological interventions for coronary heart disease: Cochrane systematic review and meta-analysis. European Journal of Heart Failure 2018;25:247-59.

\section{Rollman 2009}

Rollman BL, Belnap BH, LeMenager MS, Mazumdar S, Houck PR, Counihan PJ, et al. Telephone-delivered collaborative care for treating post-CABG depression: a randomized controlled trial. Journal of the American Medical Association 2009;302:2095-103.

\section{Romppel 2013}

Romppel M, Herrmann-Lingen C, Wachter R, Edelmann F, Düngen HD, Pieske B, et al. A short form of the General SelfEfficacy Scale (GSE-6): development, psychometric properties and validity in an intercultural non-clinical sample and a sample of patients at risk for heart failure. Psychosocial Medicaine 2013;10:Doc01.

\section{Saracutu 2018}

Saracutu M, Edwards DJ, Davies H, Rance J. Protocol for a feasibility and acceptability study using a brief ACT-based intervention for people from Southwest Wales who live with persistent pain. BMJ Open 2018;8:e021866.

\section{Schag 1984}

Schag CC, Heinrich RL, Ganz PA. Karnofsky performance status revisited: reliability, validity, and guidelines. Journal of Clinical Oncology 1984;2:187-93.

\section{Schmidt 2016}

Schmidt M, Ulrichsen SP, Pedersen L, Bøtker HE, Sørensen HT. Thirty-year trends in heart failure hospitalization and mortality rates and the prognostic impact of co-morbidity: a Danish nationwide cohort study. European Journal of Heart Failure 2016;18:490-99.

\section{Schwarzer 1995}

Schwarzer R, Jerusalem M. Generalized self-efficacy scale. Measures in Health Psychology: A User's Portfolio. Causal and Control Beliefs. Windsor, UK: NFER-NELSON, 1995.

\section{Schünemann 2019}

Schünemann HJ, Higgins JP, Vist GE, Glasziou P, Akl EA, Skoetz N, et al. Chapter 14: Completing 'Summary of findings' tables and grading the certainty of the evidence. In: Higgins JPT, Thomas J, Chandler J, Cumpston M, Li T, Page MJ, Welch VA (editors). Cochrane Handbook for Systematic Reviews of Interventions version 6.0 (updated July 2019). Cochrane, 2019. Available from www.training.cochrane.org/handbook.

\section{Sekhon 2017}

Sekhon M, Cartwright M, Francis JJ. Acceptability of healthcare interventions: an overview of reviews and development of a theoretical framework. BMC Health Service Research 2017;17:88.

\section{Seldenrijk 2015}

Seldenrijk A, Vogelzangs N, Batelaan NM, Wieman I, Van Schaik DJ, Penninx BJ. Depression, anxiety and 6-year risk of cardiovascular disease. Journal of Psychosomatic Research 2015;78:123-9.

\section{Shafie 2018}

Shafie AA, Tan YP, Ng CH. Systematic review of economic burden of heart failure. Heart Failure Reviews 2018;23:131-45.

\section{Spertus 1995}

Spertus JA, Winder JA, Dewhurst TA, Deyo RA, Prodzinski J, McDonell M, et al. Development and evaluation of the Seattle Angina Questionnaire: a new functional status measure for coronary artery disease. Journal of the American College of Cardiology 1995;25:333-41.

\section{Spielberger 1983}

Spielberger CD, Gorsuch RL, Lushene R, Vagg PR, Jacobs GA. Manual for the State-Trait Anxiety Inventory. Palo Alto, CA: Consulting Psychologists Press, 1983. 


\section{Spitzer 2006}

Spitzer RL, Kroenke K, Williams JB, Löwe B. A brief measure for assessing generalized anxiety disorder: the GAD-7. Archives of Internal Medicine 2006;166:1092-7.

\section{Staerk 2017}

Staerk L, Sherer JA, Ko D, Benjamin EJ, Helm RH. Atrial fibrillation: epidemiology, pathophysiology, and clinical outcomes. Circulation Research 2017;120:1501-17.

\section{StataCorp 2019}

StataCorp. Stata Meta-analysis Reference Manual Release 16. College Station, TX: StataCorp LLC, 2019.

\section{Sterne 2019}

Sterne JA, Savović J, Page MJ, Elbers RG, Blencowe NS, Boutron I, et al. RoB 2: a revised tool for assessing risk of bias in randomised trials. BMJ 2019;366:14898.

\section{Supervia 2019}

Supervia M, Turk-Adawi K, Lopez-Jimenez F, Pesah E, Ding R, Britto RR, et al. Nature of Cardiac Rehabilitation Around the Globe. EClinicalMedicine 2019;13:46-56.

\section{Svanborg 1994}

Svanborg P, Asberg M. A new self-rating scale for depression and anxiety states based on the Comprehensive Psychopathological Rating Scale. Acta Psychiatrica Scandinavica 1994;89:21-8.

\section{Taylor 1953}

Taylor JA. A personality scale of manifest anxiety. Journal of Abnormal and Social Psychology 1953;48:285-90.

\section{Thomas 2019}

Thomas RJ, Beatty AL, Beckie TM, Brewer LC, Brown TM, Forman DE, et al. Home-based cardiac rehabilitation: a scientific statement from the American Association of Cardiovascular and Pulmonary Rehabilitation, the American Heart Association, and the American College of Cardiology. Circulation 2019;140:e69-e89.

\section{Thompson 1998}

Thompson DR, Meadows KA, Lewin RJ. Measuring quality of life in patients with coronary heart disease. European Heart Journal 1998;19:693-5.

\section{Valenti 1996}

Valenti L, Lim L, Heller RF, Knapp J. An improved questionnaire for assessing quality of life after acute myocardial infarction. Quality of Life Research 1996;5:151-61.

\section{Van Der Kooy 2007}

Van Der Kooy K, Van Hout H, Marwijk H, Marten H, Stehouwer C, Beekman A. Depression and the risk for cardiovascular diseases: systematic review and meta-analysis. International Journal of Geriatric Psychiatry 2007;22:613-26.

\section{Wan 2014}

Wan C, Li H, Fan X, Yang R, Pan J, Chen W, et al. Development and validation of the coronary heart disease scale under the system of quality of life instruments for chronic diseases QLICDCHD: combinations of classical test theory and generalizability theory. Health and Quality of Life Outcomes 2014;12:82.

\section{Ware 1994}

Ware JE, Kosinski M, Keller SD. SF-36 Physical and Mental Health Summary Scales: a user's manual. Boston: The Health Institute, New England Medical Center, 1994.

\section{Ware 1995}

Ware JE, Kosinski M, Keller SD. SF-12: how to score the SF-12 Physical and Mental Health Summary Scales. Boston: The Health Institute, New England Medical Center, 1995.

\section{Watson 2018}

Watson W. Heart failure with preserved ejection fraction: pathologies, aetiology and directions for treatment. www.bcs.com/documents/D38_HFpEF_Review_3.pdf (accessed 15 April 2019).

\section{Whalley 2011}

Whalley B, Rees K, Davies P, Bennett P, Ebrahim S, Liu Z, et al. Psychological interventions for coronary heart disease. Cochrane Database of Systematic Reviews 2011, Issue 8. [DOI: 10.1002/14651858.CD002902]

\section{WHO 2018}

World Health Organization. Disease burden and mortality estimates by cause, age and sex, globally and by region, 2000-2016. www.who.int/healthinfo/global_burden_disease/ estimates/en/ (accessed 15 April 2019).

\section{Wiklund 1987}

Wiklund I, Lindvall K, Swedberg K, Zupkis RV. Self-assessment of quality of life in severe heart failure. An instrument for clinical use. Scandinavian Journal of Psychology 1987;28:220-5.

\section{Williams 2010}

Williams JE, Mosley TH Jr, Kop WJ, Couper DJ, Welch VL, Rosamond WD. Vital exhaustion as a risk factor for adverse cardiac events (from the Atherosclerosis Risk In Communities [ARIC] study). American Journal of Cardiology 2010;105:1661-5.

\section{Woodruffe 2015}

Woodruffe S, Neubeck L, Clark RA, Gray K, Ferry C, Finan J, et al. Australian Cardiovascular Health and Rehabilitation Association (ACRA) core components of cardiovascular disease secondary prevention and cardiac rehabilitation 2014. Heart Lung and Circulation 2015;24:430-41.

\section{Zigmond 1983}

Zigmond AS, Snaith RP. The Hospital Anxiety and Depression Scale. Acta Psychiatrica Scandinavica 1983;67:361-70.

\section{Zung 1965}

Zung WW. A self-rating scale for depression. Archives of General Psychiatry 1965;12:63-70. 
ADDITIONAL TABLES

Table 1. Intervention versus control for the treatment of depression or anxiety, or both

\section{Intervention versus control for the treatment of depression or anxiety, or both}

Patient or population: people with heart disease

Setting: hospital or community settings

Intervention: psychological intervention

Comparison: usual care

\begin{tabular}{|c|c|c|c|c|c|c|}
\hline \multirow[t]{2}{*}{ Outcomes } & \multicolumn{2}{|c|}{$\begin{array}{l}\text { Anticipated absolute effects }{ }^{*} \\
(95 \% \mathrm{Cl})\end{array}$} & \multirow{2}{*}{$\begin{array}{l}\text { Relative } \\
\text { effect } \\
(95 \% \mathrm{CI})\end{array}$} & \multirow{2}{*}{$\begin{array}{l}\text { № of par- } \\
\text { ticipants } \\
\text { (trials) }\end{array}$} & \multirow{2}{*}{$\begin{array}{l}\text { Certainty } \\
\text { of the evi- } \\
\text { dence } \\
\text { (GRADE) }\end{array}$} & \multirow[t]{2}{*}{$\begin{array}{l}\text { Com- } \\
\text { ments }\end{array}$} \\
\hline & $\begin{array}{l}\text { Risk with } \\
\text { control }\end{array}$ & $\begin{array}{l}\text { Risk with } \\
\text { treatment }\end{array}$ & & & & \\
\hline
\end{tabular}

\section{Depression}

\section{Anxiety}

HRQOL

\section{All-cause mortality}

\section{Cardiovascular mortality}

\section{Cardiovascular hospitalisations}

\section{Cost effectiveness}

*The risk in the intervention group (and its 95\% confidence interval) is based on the assumed risk in the comparison group and the relative effect of the intervention (and its $95 \% \mathrm{Cl}$ ).

CI: confidence interval; HRQoL: health-related quality of life; $\mathbf{R C T}$ : randomised controlled trial; RR: risk ratio

\section{GRADE Working Group grades of evidence}

High certainty: we are very confident that the true effect lies close to that of the estimate of the effect.

Moderate certainty: we are moderately confident in the effect estimate: the true effect is likely to be close to the estimate of the effect, but there is a possibility that it is substantially different.

Low certainty: our confidence in the effect estimate is limited: the true effect may be substantially different from the estimate of the effect.

Very low certainty: we have very little confidence in the effect estimate: the true effect is likely to be substantially different from the estimate of effect.

\section{AP PEN DICES}

\section{Appendix 1. Preliminary MEDLINE (Ovid) search strategy}

1 exp Heart Diseases/

2 (heart adj4 disease $\left.{ }^{\star}\right)$.tw.

3 exp Coronary Disease/

4 (coronary adj2 disease*).tw.

5 CHD.tw.

Psychological interventions for depression and anxiety in patients with coronary heart disease, heart failure or atrial fibrillation 
6 exp Heart Failure/

7 (heart adj6 fail $\left.{ }^{\star}\right)$. tw.

8 exp Atrial Fibrillation/

9 Atrial fibrillation.tw.

10 exp Myocardial Ischemia/

11 (myocard $^{\star}$ adj4 (ischaemi* or ischemi $\left.\left.{ }^{\star}\right)\right)$. tw.

12 ((ischaemi ${ }^{\star}$ or ischemi $\left.{ }^{\star}\right)$ adj4 heart).tw.

13 exp Myocardial Infarction/

14 (myocard $^{*}$ adj4 infarct $\left.{ }^{\star}\right)$. tw.

15 (heart adj4 (infarct* or attack $\left.{ }^{\star}\right)$ ).tw.

16 exp Angina Pectoris/

17 angina.tw.

18 stenocardia*.tw.

19 exp Percutaneous Coronary Intervention/

20 (percutaneous coronary adj2 (interven* or revascular $\left.{ }^{\star}\right)$ ).tw.

21 (pci or ptca).tw.

22 exp Coronary Artery Bypass/

23 (coronary adj4 bypass $\left.{ }^{\star}\right)$. tw.

24 CABG.tw.

25 aortocoronary bypass ${ }^{\star}$. tw.

26 Heart Bypass, Left/ or Heart Bypass, Right/

27 exp Myocardial Revascularization/

28 myocard $^{\star}$. tw.

29 cardiac $^{*}$. tw.

30 exp Angioplasty/

31 angioplast ${ }^{\star}$. tw.

32 ((coronary or arterial) adj4 dilat*).tw.

33 endoluminal repair ${ }^{\star}$.tw.

34 exp Stents/

35 stent $^{\star}$. tw.

$36 \exp$ Atherectomy/

37 atherectom*.tw.

38 acute coronary syndrom ${ }^{\star} . t w$.

391 or 2 or 3 or 4 or 5 or 6 or 7 or 8 or 9 or 10 or 11 or 12 or 13 or 14 or 15 or 16 or 17 or 18 or 19 or 20 or 21 or 22 or 23 or 24 or 25 or 26 or 27 or 28 or 29 or 30 or 31 or 32 or 33 or 34 or 35 or 36 or 37 or 38

Psychological interventions for depression and anxiety in patients with coronary heart disease, heart failure or atrial fibrillation 
40 exp Psychotherapy/

41 psychotherap*.tw.

42 ((psycholog* or psychosocial) adj intervent $\left.{ }^{\star}\right)$.tw.

43 exp Relaxation Techniques/

44 Relaxation therapy/

45 relax$^{\star}$. tw.

46 exp Counseling/

47 (counselling or counseling).tw.

48 Stress, Psychological/

49 (stress adj4 (management or reduc $\left.{ }^{\star}\right)$ ).tw.

50 Autogenic Training/

51 autogenic ${ }^{\star} . t w$.

52 Meditation/

53 meditat ${ }^{\star}$. tw.

54 Mindfulness/

55 Mindfulness.tw.

56 Cognitive Behavioral Therapy/

57 (cognitive adj4 (therap* or technique $\left.{ }^{\star}\right)$ ).tw.

\section{CBT.tw.}

59 ((behavior* or behaviour $\left.^{\star}\right)$ adj4 (modify or modificat ${ }^{\star}$ or therap* or change)).tw.

$60\left(\right.$ manage ${ }^{\star}$ adj2 (anxiety or depres $\left.\left.{ }^{\star}\right)\right)$. tw.

61 Psychopathology/

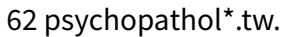

63 distress ${ }^{\star} . t w$.

64 hypnotherap*.tw.

65 (goal $^{\star}$ adj3 setting).tw.

66 (psycho-educat $^{\star}$ or psychoeducat ${ }^{\star}$ ).tw.

67 (motivat $^{\star}$ adj3 interv $\left.{ }^{\star}\right)$. tw.

6840 or 41 or 42 or 43 or 44 or 45 or 46 or 47 or 48 or 49 or 50 or 51 or 52 or 53 or 54 or 55 or 56 or 57 or 58 or 59 or 60 or 61 or 62 or 63 or 64 or 65 or 66 or 67

6939 and 68

70 randomized controlled trial.pt.

71 controlled clinical trial.pt.

72 randomized.ab.

73 placebo.ab.

Psychological interventions for depression and anxiety in patients with coronary heart disease, heart failure or atrial fibrillation 
74 clinical trials as topic.sh.

75 randomly.ab.

76 trial.ti.

7770 or 71 or 72 or 73 or 74 or 75 or 76

78 exp animals/ not humans.sh.

7977 not 78

8069 and 79

81 limit 80 to $y r=" 2009-c u r r e n t "$

\section{CONTRIBUTIONS OF AUTHORS}

CFS drafted the initial protocol. All review authors have revised and contributed to the drafting of the protocol, and all have approved the final version of the protocol for publication.

\section{DECLARATIONS OF INTEREST}

CFS: no known conflict of interest RST: no known conflict of interest KMG: no known conflict of interest JDL: no known conflict of interest DRT: no known conflict of interest SHR is first author for a clinical trial (HTA CADENCE study) that is likely to satisfy the eligibility criteria and be included in this review.

\section{SOURCES OF SUPPORT}

\section{Internal sources}

- No sources of support supplied

\section{External sources}

- This project was supported by the National Institute for Health Research, via Cochrane Infrastructure funding to Cochrane Heart. The views and opinions expressed therein are those of the authors and do not necessarily reflect those of the Systematic Reviews Programme, NIHR, NHS or the Department of Health, UK. 